\title{
Pseudo-dielectric relaxation-enabled electrothermal tuning of reflective color in a soft chiral photonic crystal
}

Po-Chang Wu, Guan-Wei Wu, Wei Lee

Po-Chang Wu, Guan-Wei Wu, Wei Lee, "Pseudo-dielectric relaxation-enabled electrothermal tuning of reflective color in a soft chiral photonic crystal," Proc. SPIE 11092, Liquid Crystals XXIII, 1109202 (29 August 2019); doi: 10.1117/12.2526099

Event: SPIE Organic Photonics + Electronics, 2019, San Diego, California, United States 


\title{
Pseudo-dielectric relaxation-enabled electrothermal tuning of reflective color in a soft chiral photonic crystal
}

\author{
Po-Chang $\mathrm{Wu}^{\mathrm{a}}$, Guan-Wei $\mathrm{Wu}^{\mathrm{b}}$, and Wei Lee ${ }^{\mathrm{a}, *}$ \\ ${ }^{a}$ Institute of Imaging and Biomedical Photonics, College of Photonics, National Chiao Tung \\ University, Guiren Dist., Tainan 71150, Taiwan \\ ${ }^{\mathrm{b}}$ Institute of Lighting and Energy Photonics, College of Photonics, National Chiao Tung University, \\ Guiren Dist., Tainan 71150, Taiwan
}

\begin{abstract}
Many published papers bear flaw information caused by misinterpreting the pseudo-dielectric relaxation in liquid crystal (LC) confined in thin cells. The pseudo-dielectric relaxation stems from the cell geometries or parameters, which should not be misrecognized as an inherent material property of the LC. In this work, we explored the pseudo-dielectric relaxation and demonstrated an unconventional tuning means to electrically manipulate the reflective color of a typical cholesteric LC cell based on the heating due to the pseudo-dielectric relaxation. A tuning range in the full-color spectrum can be easily achieved by a limited temperature change of merely a few degrees Celsius.
\end{abstract}

Keywords: Cholesteric liquid crystals, Color tuning, Pseudo-dielectric relaxation, Pseudo-dielectric heating

\section{INTRODUCTION}

A cholesteric liquid crystal (CLC) cell in the Grandjean planar state revealing uniform standing helix with helical axis perpendicular to the substrate plane is alluring for the existence of Bragg's reflection bandgap in the optical spectrum that enables the reflection of circularly polarized light with the same handedness as that of the helix. Owing to the uniqueness, CLC cells with helical pitch length $(p)$ comparable to the optical wavelength have widely been exploited as promising soft chiral photonic crystals for many applications, such as reflectors, filters, lasers, displays, and biosensors. ${ }^{1-6}$ Based on the Bragg law, the reflection bandgap of a CLC is centered at the wavelength of $\lambda_{\mathrm{c}}=n \times P$ where $n=\left(n_{\mathrm{e}}+n_{\mathrm{o}}\right) / 2$ is the average of the ordinary $\left(n_{\mathrm{o}}\right)$ and extraordinary $\left(n_{\mathrm{e}}\right)$ refractive indices of the CLC material. When a CLC is made by doping chiral additives into an achiral nematic host, the magnitude of $P$ can be preliminarily determined by the helical twisting power (HTP) of the chiral dopant and the dopant concentration $(c)$, following the relation of $P=(\operatorname{HTP} \times c)^{-1}$. Because of the widespread application of LCs for electro-optical devices, applying electric fields to manipulate $\lambda_{\mathrm{c}}$ of the CLC bandgap has long been a major trend for the revolutionary development of CLC devices with flexibly tunable optical features. In this regard, electrical tuning in $\lambda_{\mathrm{c}}$ based on the use of a conventional CLC with negative dielectric anisotropy $(\Delta \varepsilon<0)$ has been implemented by direct-current (DC) voltages via the induction of electrohydrodynamic instability, ${ }^{7}$ electromechanical cell-gap bending ${ }^{8}$ or defect annealing, ${ }^{9}$ or by alternating-current (AC) voltages via the incorporation of ferroelectric LCs as the dopant added in the $\mathrm{CLC}^{10}$ or as the commander coated on the substrates. ${ }^{11}$ However, the tunable $\lambda_{\mathrm{c}}$ ranges of these electrical approaches are quite limited $(<100 \mathrm{~nm})$ and the operating voltages in either the DC or AC term are too high $(V$ $>100 \mathrm{~V}$ ) to make them of practice. Differently, Xiang et al. have shown electrical $\lambda_{\mathrm{c}}$ tuning in a wide wavelength range covering the ultraviolet, visible and infrared spectral regions in a so-called oblique helicoid state of an unconventional LCs consisting of a rod-like CLC doped with bent-core mesogenic molecules CB7CB and CB6OCB. ${ }^{12}$ Apart from the electrical tuning, doping a proper amount of left-handed chiral S-811 into an achiral nematic LC to make a CLC with the SmA-CLC phase transition has been proven as one of feasible pathways to modulating $\lambda_{c}$ via the thermal variation. ${ }^{9}{ }^{13-16}$ This temperature-varying wavelength behavior can be nicely fitted by Keating's theory ${ }^{13,14}$ and has been experimentally examined in terms of the temperature-dependent twist-elastic constant and the pitch near the SmA-CLC transition ${ }^{15}$ or the dopant solubility. ${ }^{16}$ Other approaches aiming to achieve thermally tunable $\lambda_{\mathrm{c}}$ have also been attained by the incorporation of unconventional bent-core LCs with the twist-bend phase ${ }^{17}$ or specific chiral additive with thermally sensitive HTP into a conventional CLC. ${ }^{18}$ On the other hand, Wang et al. proposed to disperse mesogen-grafted gold nanorods into the CLC

*wlee@nctu.edu.tw; phone +886 (0)6 303-2121; fax +886 (0)6 303-2535 
with thermosensitivity of the helical superstructure and complied near-infrared-directed helical inversion and nearinfrared-driven bandgap shift via the photothermal effect. ${ }^{19}$ Hsiao et al. made a dual-frequency (DF) CLC mixture with strong temperature-dependent $\lambda_{c}$, which red-shifts with increasing temperature, by doping a thermosensitive chiral dopant together with a well-known chiral agent R-5011 (Merck) into the DFLC. By subjecting AC voltages at frequencies beyond the crossover frequency, the dielectric anisotropy of the DFCLC becomes negative and $\lambda_{\mathrm{c}}$ can be electrically controlled by modulating the frequency to induce temperature variation via dielectric heating. ${ }^{20}$

In view of current LC technologies, a LC for electro-optic applications is commonly enclosed in between a pair of glass substrates with inner surfaces being coated with indium-tin oxide (ITO) as the electrode material. Because ITO has finite conductivity, this sandwich-type cell geometry can be regarded as equivalent to an electric circuit consisting of a capacitor from the $\mathrm{LC}$ layer $\left(C_{\mathrm{LC}}\right)$ and a resistor from the electrode $\left(R_{\mathrm{ITO}}\right)$ in series. Owing to the induction of losses from the $R_{\mathrm{ITO}} C_{\mathrm{LC}}$ circuit, a pseudo-dielectric relaxation is unavoidably induced in the complex dielectric spectrum and the corresponding pseudo-dielectric relaxation frequency $\left(f_{\mathrm{PR}}\right)$ has been formulated in terms of $R_{\mathrm{ITO}}$, the cell gap $(d)$, the electrode area $(A)$, and the low-frequency-limit dielectric permittivity of the LC material $\left(\varepsilon_{\mathrm{s}}\right)$ according to $^{21}$ :

$$
f_{\mathrm{PR}}=\frac{1}{2 \pi R_{\mathrm{ITO}} C_{\mathrm{LC}}}=\frac{1}{2 \pi R_{\mathrm{ITO}}} \frac{d}{\varepsilon_{0} \varepsilon_{\mathrm{s}} A},
$$

where $\varepsilon_{0}\left(=8.854 \times 10^{-12} \mathrm{~F} \cdot \mathrm{m}^{-1}\right)$ represents the permittivity in free space. As reported by relevant studies, this pseudodielectric relaxation would suppress or combine with other dielectric behaviors, leading to the increase in complexity for dielectric investigations and interpretations. For this reason, except for the use of a lower-resistivity electrode material, say, a metal, instead of a metal oxide and of cells of much thicker cell gaps, ${ }^{22-24}$ several mathematical methods have been suggested in attempt to exclude this unwanted dielectric signal. ${ }^{21,}{ }^{25}$ In this keynote article, we report on our experimental discovery of the pseudo-dielectric relaxation that induces dielectric-like heating in a conventional LC cell subject to an external AC voltage. Interestingly, although the observed pseudo-dielectric heating behavior is quite different from the well-known dielectric heating effect in DFLCs, its exact mechanism can be theoretically interpreted by properly modifying the simplified model established by Schadt. ${ }^{22}$ Furthermore, we applied this pseudo-dielectric heating effect to a thermally sensitive $-\Delta \varepsilon$ CLC cell and proposed a novel electro-thermal technique for $\lambda_{c}$ tuning. It is worth mentioning that both the tunable $\lambda_{\mathrm{c}}$ range and the operating voltage required are much superior to those of existing electric tuning techniques. For example, $\lambda_{\mathrm{c}}$ can be directly and reversibly modulated within the entire visible regime (i.e., from $\sim 760 \mathrm{~nm}$ to $\sim 380 \mathrm{~nm}$ ) by varying the amplitude of the applied voltage in the range between $3.5 \mathrm{~V}_{\text {rms }}$ and $12 \mathrm{~V}_{\text {rms }}$ at a fixed frequency of $2 \mathrm{MHz}$ or by varying the frequency in the range between $1 \mathrm{kHz}$ and $1.3 \mathrm{MHz}$ at a fixed amplitude of $12 \mathrm{~V}_{\mathrm{rms}}{ }^{26}$

\section{EXPERIMENTAL}

The chiral additive and the LC host (both purchased from Merck) for the preparation of CLC mixtures under investigations are, respectively, S-811 with left-handed chirality and MLC-6608 with physical properties (adopted from the Merck datasheet) of clearing temperature $T_{\mathrm{c}}=91{ }^{\circ} \mathrm{C}$, dielectric anisotropy $\Delta \varepsilon=-4.2$, and birefringence $\Delta n=0.083$. After stirring the mixtures containing various chiral dopant contents at the temperature of $\sim 100{ }^{\circ} \mathrm{C}$ for two hours, the CLCs were injected into identical planar-aligned empty cells with ITO as the electrodes and the cell parameters are $d=5.0 \pm 0.5$ $\mu \mathrm{m}, A=1.0 \mathrm{~cm}^{2}$, and $R_{\text {Iто }}=310 \Omega$. As indicated previously, when the chiral dopant concentration is high enough such that the SmA phase can be induced at a lowered temperature below the CLC phase, one would expect this kind of CLC mixture to exhibit strong temperature dependence of $\lambda_{\mathrm{c}}$ near the phase transition temperature $\left(T_{\mathrm{SmA}}\right) .{ }^{9-11}$ Following this rule, we preliminarily examined the phase sequences of the samples in the cooling process by means of temperaturedependent dielectric spectroscopy and textural observations so as to find an optimal S-811 content in MLC-6608 that enables the CLC to reveal $T_{\mathrm{SmA}}$ at near room temperature for ease of operation. As summarized in Fig. 1, the cell with 30wt\% S-811 upon decreasing temperature exhibits phase transitions from the isotropic to blue phase (BP) at $T_{\mathrm{BP}}=58{ }^{\circ} \mathrm{C}$ and from BP to CLC at $T_{\mathrm{CLC}}=47^{\circ} \mathrm{C}$ but the SmA phase is absent even when the temperature is as low as $0{ }^{\circ} \mathrm{C}$. Increasing the S-811 concentration results in the decrease in $T_{\mathrm{BP}}\left(T_{\mathrm{CLC}}\right)$ to $44{ }^{\circ} \mathrm{C}\left(30^{\circ} \mathrm{C}\right)$ at $40 \mathrm{wt} \%$ and $39^{\circ} \mathrm{C}\left(27^{\circ} \mathrm{C}\right)$ at $45 \mathrm{wt} \%$. Notably, the SmA phase is detected at $T_{\mathrm{SmA}}=12{ }^{\circ} \mathrm{C}$ and $29^{\circ} \mathrm{C}$ in the cells with 40 -wt\% and 45 -wt $\% \mathrm{~S}-811$, respectively. Once the content of S-811 reaches $50 \mathrm{wt} \%$, neither BP nor CLC is observed, leading to the direct transition from the isotropic to SmA phase at $T_{\mathrm{SmA}}=29^{\circ} \mathrm{C}$. 


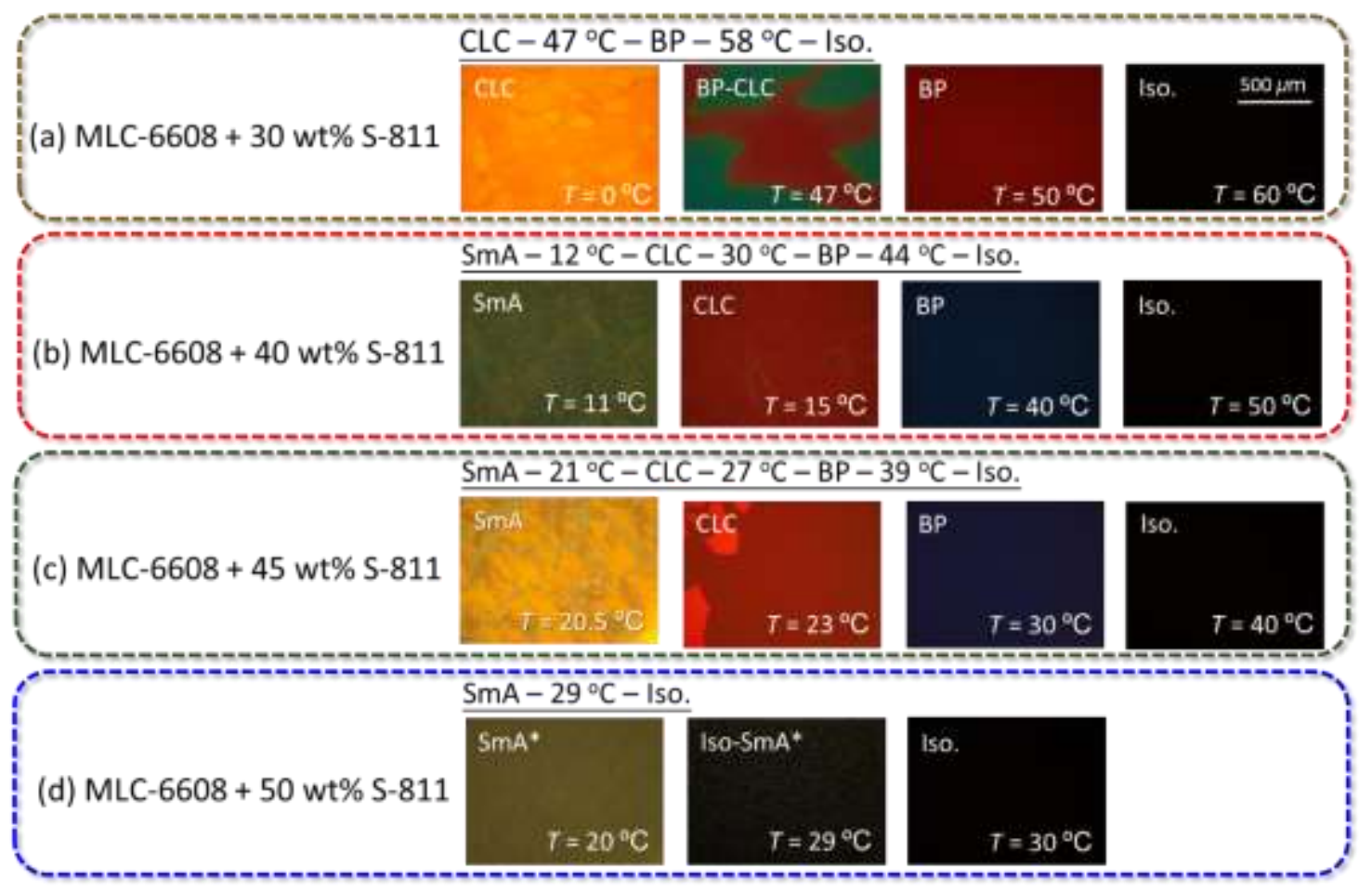

Figure 1. Phase transition sequences determined by means of temperature-dependent dielectric spectroscopy and polarizing optical microscopy for reflective optical textures at specific temperatures of CLCs with the $-\Delta \varepsilon$ nematic host MLC-6608 doped with (a) 30-wt\%, (b) 40-wt\%, (c) 45-wt\%, and (d) 50-wt $\%$ S-811.

According to the results given in Fig. 1, the CLC containing $45 \mathrm{wt} \% \mathrm{~S}-811$ with $T_{\mathrm{SmA}}=21^{\circ} \mathrm{C}$ was primarily used for further optical, dielectric and electro-thermal investigations in this study. Note that the phase sequence of the cell with 45 wt $\% \mathrm{~S}-811$ is SmA-2 $1{ }^{\circ} \mathrm{C}-\mathrm{CLC}-39{ }^{\circ} \mathrm{C}-$ Iso in the heating process; namely, $T_{\mathrm{SmA}}$ is invariant but the BP disappears and $T_{\mathrm{BP}}$ is replaced by $T_{\mathrm{CLC}}$ in comparison with the cooling process. This phenomenon is seen in other samples with distinct $\mathrm{S}$ 811 concentrations.

Optical textures of the cells were acquired using a polarizing optical microscope (Olympus BX51) operating in the reflection mode. The value of $\lambda_{\mathrm{c}}$ was deduced from the reflection bandgap in the transmission spectrum as measured by a fiber-optic spectrometer (Ocean Optics HR2000+) in conjunction with a halogen light source (Ocean Optics HL2000). An LCR meter (Agilent E4980A) was exploited to measure the complex dielectric spectra. It also served as a function generator, supplying AC voltages in the sinusoidal waveform with controllable amplitudes (from $0.05 \mathrm{~V}_{\text {rms }}$ to $15 \mathrm{~V}_{\text {rms }}$ ) and frequencies (from $20 \mathrm{~Hz}$ to $2 \mathrm{MHz}$ ). An IR camera (FLIR ThermaCam ${ }^{\circledR} \mathrm{P} 25$ ) was employed to monitor the cell temperature and, in turn, to quantify the magnitude of temperature increase, derived from the voltage-induced pseudo-dielectric heating. Specific for the investigations of the pseudo-dielectric heating effect and electro-thermal properties, relevant measurements were performed in a stable thermal environment at a fixed ambient temperature of $\sim 23{ }^{\circ} \mathrm{C}$ (i.e., near $T_{\mathrm{SmA}}=21{ }^{\circ} \mathrm{C}$ ), with insufficient thermal dissipations at the interface and constant humidity as well as air flow. This was achieved by enclosing the cell in a container $\left(169 \times 110 \times 25.8 \mathrm{~mm}^{3}\right)$ with air as the surrounding medium.

\section{RESULTS AND DISCUSSION}

\subsection{Voltage induction of pseudo-dielectric heating}

This section is aimed to experimentally clarify the pseudo-dielectric relaxation-enabled electrical heating effect and theoretically elucidate the exact mechanism by a model. Figure 2(a) depicts the real- $\left(\varepsilon^{\prime}\right)$ and imaginary- $\left(\varepsilon^{\prime \prime}\right)$ part dielectric spectra of a CLC cell applied with a probe voltage of $V=0.5 \mathrm{~V}_{\text {rns }}$ at $T=23{ }^{\circ} \mathrm{C}$. Normally, the dielectric permittivity at the high-frequency limit $\left(\varepsilon_{\mathrm{H}}\right)$ of a dielectric medium driven by an electric field must be larger than unity due to the non-zero 


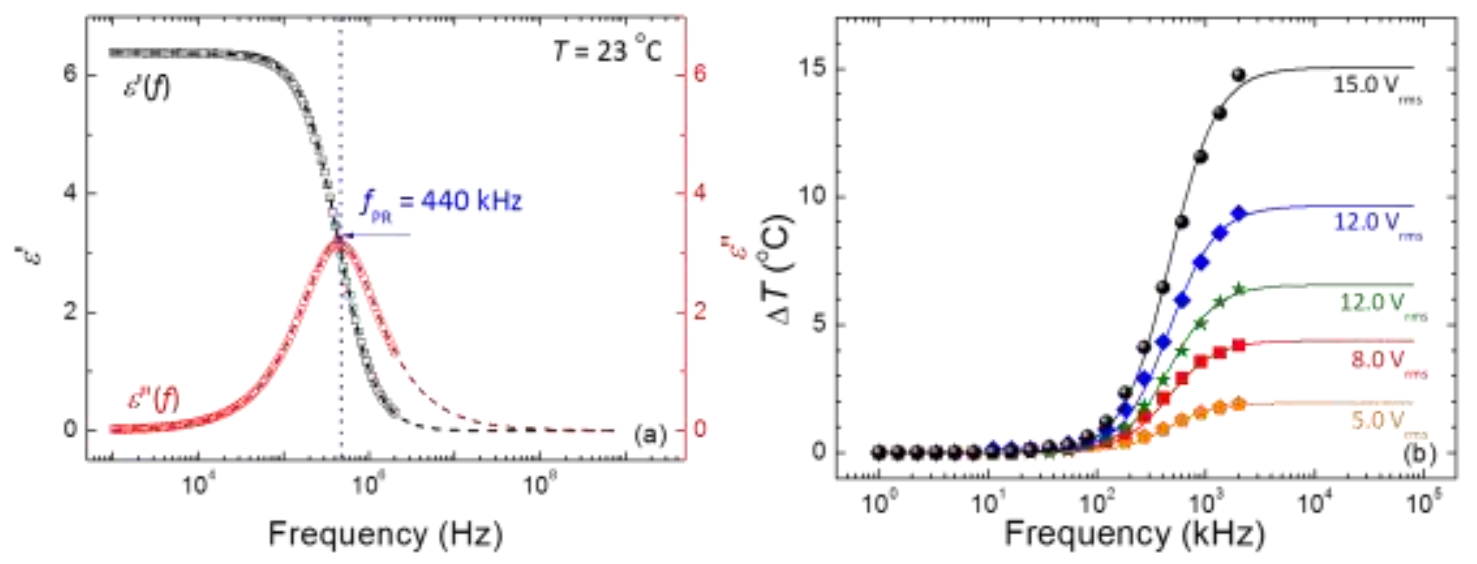

Figure 2. Experimental and simulation results of (a) complex dielectric spectra of the CLC cell and (b) frequency dependence of temperature elevations $(f-\Delta T)$ at various voltage amplitudes. Here simulated curves in (a) and (b) are plotted based on Eqs. (2) and (3), respectively.

electric susceptibility, except for the case of the vacuum. Therefore, the observed dielectric relaxation in the measured frequency range between $1 \mathrm{kHz}$ and $2 \mathrm{MHz}$ with $\varepsilon_{\mathrm{H}} \sim 0$ is not the case from any polarization behavior of the LC bulk but is the pseudo-dielectric relaxation characterized by "dielectric" losses from the sandwich-type cell geometry. By fitting the complex dielectric spectrum $\left(\varepsilon^{*}(f)\right)$ into the Debye equation as follows:

$$
\varepsilon^{*}(f)=\varepsilon_{\mathrm{H}}+\frac{\left(\varepsilon_{\mathrm{s}}-\varepsilon_{\mathrm{H}}\right)}{1+i\left(f / f_{\mathrm{PR}}\right)},
$$

one can get linear increase in $f_{\mathrm{PR}}$ from $440 \mathrm{kHz}$ (at $T=23^{\circ} \mathrm{C}$ ) to $473 \mathrm{kHz}$ (at $T=38^{\circ} \mathrm{C}$ ) due to the variation of LC molecules and thus the value of $\varepsilon_{\mathrm{s}}$ with the temperature. The integrity of the pseudo-dielectric relaxation, plotted by substituting the parameters of $\varepsilon_{\mathrm{s}}=6.4, \varepsilon_{\mathrm{H}}=0$ and $f_{\mathrm{PR}}=440 \mathrm{kHz}$ into Eq. (2) (dashed lines in Fig. 2(a)), is in good agreement with the experimental data. Figure 2(b) shows the frequency-dependent temperature elevations of the cell driven by various voltage amplitudes. Here, the cell temperature in the field-off state is $23^{\circ} \mathrm{C}$, meaning that the temperature increase $\Delta T$ is equal to $T-23^{\circ} \mathrm{C}>0$. Each data point was measured by an IR camera after applying the voltage for $480 \mathrm{~s}$ so as to ensure the cell temperature to be in the steady state. When the frequency is higher than an onset value $\left(f_{0}\right)$, the cell temperature starts to rise with increasing voltage frequency at a constant amplitude or with increasing amplitude at a fixed frequency. For example, $\Delta T$ of the cell driven by $2 \mathrm{MHz}$ voltages is $1.9^{\circ} \mathrm{C}$ (i.e., $T=24.9^{\circ} \mathrm{C}$ ) at $V=5 \mathrm{~V}_{\text {rms }}$ and it increases to $14.8^{\circ} \mathrm{C}$ (i.e., $T=37.8^{\circ} \mathrm{C}$ ) at $V=15 \mathrm{~V}_{\mathrm{rms}}$. According to the correlation between the heat generation and the dielectric features of a $\mathrm{LC}$ cell, one can modify Schadt's model ${ }^{22}$ with the condition of $\varepsilon_{\mathrm{H}}=0$ and attempt to explain the results of Fig. 2(b) in terms of the voltage-induced pseudo-dielectric heating by the following equation:

$$
\Delta T=\left(\frac{V^{2} A \varepsilon_{0} \varepsilon_{\mathrm{s}}}{d h}\right)\left(\frac{2 \pi f^{2} / f_{\mathrm{R}}}{1+\left(f / f_{\mathrm{R}}\right)^{2}}\right),
$$

where $h$ is the specific heat conductivity of the cell and $f_{\mathrm{R}}$ is the relaxation frequency of the dominated pseudo-dielectric relaxation for the pseudo-dielectric heating. It is worth mentioning that all $f-\Delta T$ curves as shown in Fig. 2(b) can nicely be fitted by Eq. (3), yielding the corresponding coefficients of determination $R^{2}=0.99$ and the fitting parameters $h$ and $f_{\mathrm{R}}$ deduced as listed in Table 1. Averagely, the value of $f_{\mathrm{R}}$ is $\sim 456.7 \pm 12 \mathrm{kHz}$ (with $h \sim 0.046 \pm 0.005 \mathrm{~W} \cdot \mathrm{K}^{-1}$ ), which lies in the range between $f_{\mathrm{PR}}=440 \mathrm{kHz}$ at $T=23^{\circ} \mathrm{C}$ and $f_{\mathrm{PR}}=473 \mathrm{kHz}$ at $T=38^{\circ} \mathrm{C}$. The results undoubtedly confirm the dominant heating induced by the pseudo-dielectric relaxation. The possible reasons leading to the variation of $f_{\mathrm{R}}$ with the applied voltage would be systematical errors from measurements by the IR camera and the voltage-dependent $f_{0}$ as well as the temperature-dependent $f_{\mathrm{PR}}$. By substituting the cell parameters (i.e. $A=1 \mathrm{~cm}^{2}, d=5 \mu \mathrm{m}$, and $\varepsilon_{\mathrm{s}}=6.4$ ) and fitting parameters (i.e., $f_{\mathrm{R}}$ and $h$ listed in Table 1) into Eq. (3), it can be recognized from the simulated $f-\Delta T$ curves (solid lines in Fig. 2(b)) in the frequency regime between $1 \mathrm{kHz}$ and $80 \mathrm{MHz}$ that all $\Delta T$ eventually reach saturated values $\left(\Delta T_{\max }\right)$ at higher frequencies (i.e., $f>10 \mathrm{MHz}$ in Fig. 2(b)). In considering the condition of $f \gg>f_{\mathrm{R}}$ to simplify Eq. (3), this can be reasonably explained by 
Table 1. Specific heat conductivity $(h)$ and relaxation frequency $\left(f_{\mathrm{R}}\right)$, deduced by fitting the $f-\Delta T$ data in Fig. 2(b) using Eq. (3), and simulated and calculated maximal values of the voltage-induced temperature elevations $\left(\Delta T_{\max }\right)$ acquired in accordance with the simulation curves in Fig. 2(b) and using Eq. (5), respectively.

\begin{tabular}{ccccc}
\hline \hline$V\left(\mathrm{~V}_{\text {rms }}\right)$ & $h\left(\mathrm{~W} \cdot \mathrm{K}^{-1}\right)$ & $f_{\mathrm{R}}(\mathrm{kHz})$ & $\begin{array}{c}\Delta T_{\max }\left({ }^{\circ} \mathrm{C}\right) \\
\text { (simulation) }\end{array}$ & $\begin{array}{c}\Delta T_{\max }\left({ }^{\circ} \mathrm{C}\right) \\
(\text { calculation })\end{array}$ \\
\hline 5 & 0.041 & 443.7 & 1.95 & 1.97 \\
8 & 0.047 & 452.0 & 4.38 & 4.39 \\
10 & 0.050 & 459.0 & 6.56 & 6.45 \\
12 & 0.049 & 461.0 & 9.64 & 9.48 \\
15 & 0.050 & 468.0 & 15.1 & 14.52 \\
\hline \hline
\end{tabular}

$\Delta T\left(f>>f_{\mathrm{R}}\right)=2 \pi f_{\mathrm{R}}\left(\frac{V^{2} A \varepsilon_{0} \varepsilon_{\mathrm{s}}}{d h}\right)$.

Interestingly, if we substitute Eq. (1) into Eq. (4) by setting $f_{\mathrm{R}}=f_{\mathrm{PR}}$, the value of $\Delta T_{\max }$ can further be formulated by

$$
\Delta T_{\text {max }} \approx \frac{V^{2}}{h R_{\text {ITO }}} .
$$

Equation (5) specifies that the maximal steady-state temperature change is inversely proportional the cell resistivity and linearly varies with the square of the voltage amplitude. Specific for the proposed pseudo-dielectric heating effect, Eq. (5) is indeed valid in that the calculated values of $\Delta T_{\max }$ from this equation of the CLC cell at five voltage conditions are nearly identical to those obtained by simulations, as summarized in Table 1 . As a result, we refer to the pseudo-dielectric relaxation from the cell geometry with finite electrode conductivity and successfully demonstrate a new "dielectric" heating behavior whose mechanism is different from that of the well-known one involving the molecular rotations in DFLCs.

\subsection{Electro-thermal tunability of $\lambda_{c}$ and reflective colors}

For the investigated CLC cell (i.e., MLC-6608 + 45-wt\% S-811), we have experimentally confirmed that the value of $\lambda_{\mathrm{c}}$ is very sensitive to the temperature, showing blueshift of the CLC bandgap with increasing temperature, from $\lambda_{\mathrm{c}} \sim 760 \mathrm{~nm}$ at $T=23{ }^{\circ} \mathrm{C}$ to $\lambda_{\mathrm{c}} \sim 380 \mathrm{~nm}$ at $T=32{ }^{\circ} \mathrm{C} .{ }^{26,27}$ By simulating the $T-\lambda_{\mathrm{c}}$ curves using Keating's formula
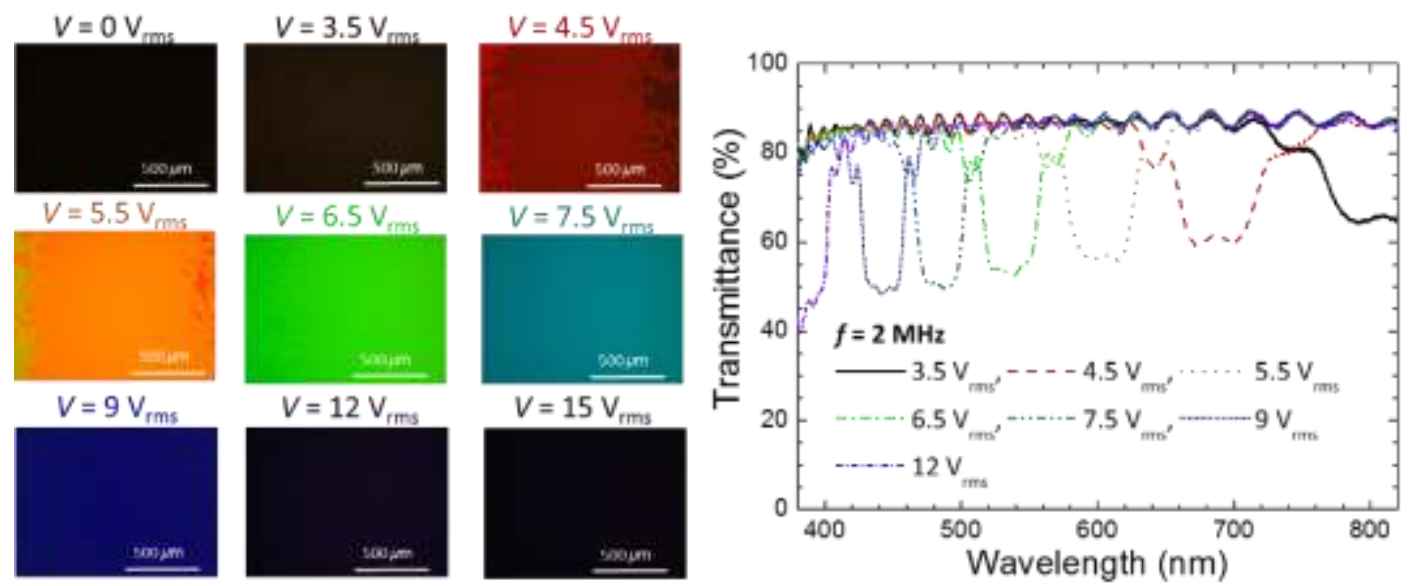

Figure 3. Reflective optical textures and transmission spectra of the CLC cell driven by various voltages at $2 \mathrm{MHz}$. The temperature of the cell in the voltage off state is $T=22^{\circ} \mathrm{C}$. 

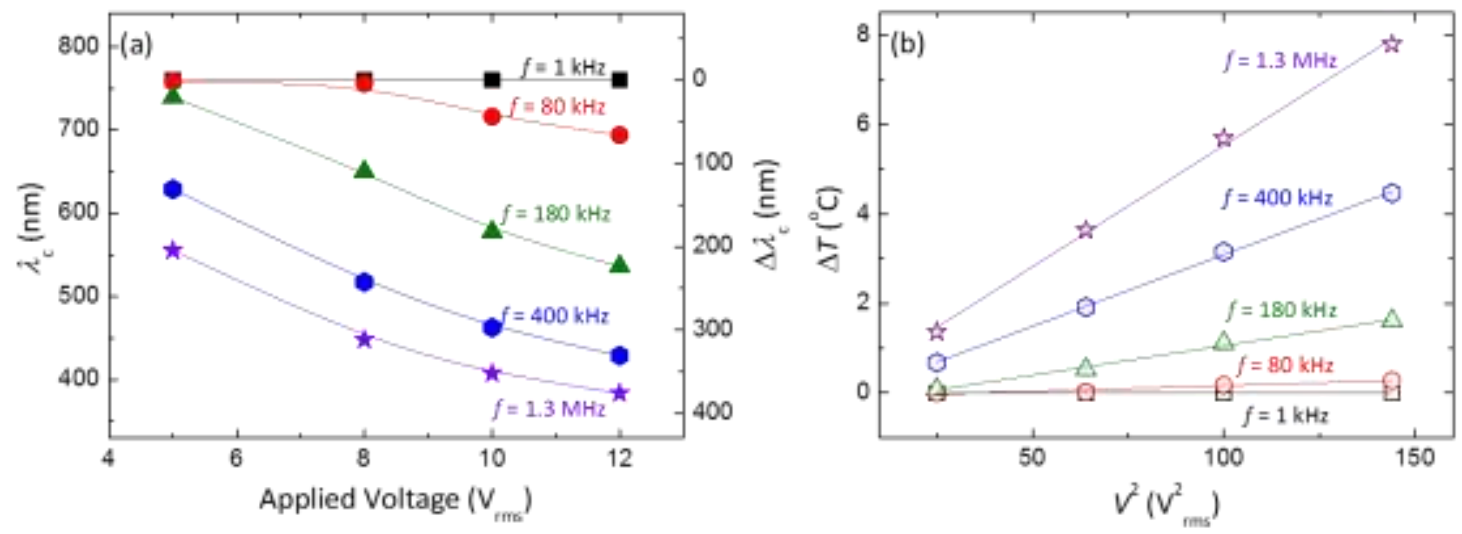

Figure 4. (a) Voltage dependence of $\lambda_{\mathrm{c}}\left(\Delta \lambda_{\mathrm{c}}\right)$ and (b) the linear correlation between $\Delta T$ and $V^{2}$ of the CLC cell at various frequency conditions. The temperature of the cell in the voltage off state is $T=23{ }^{\circ} \mathrm{C}$.
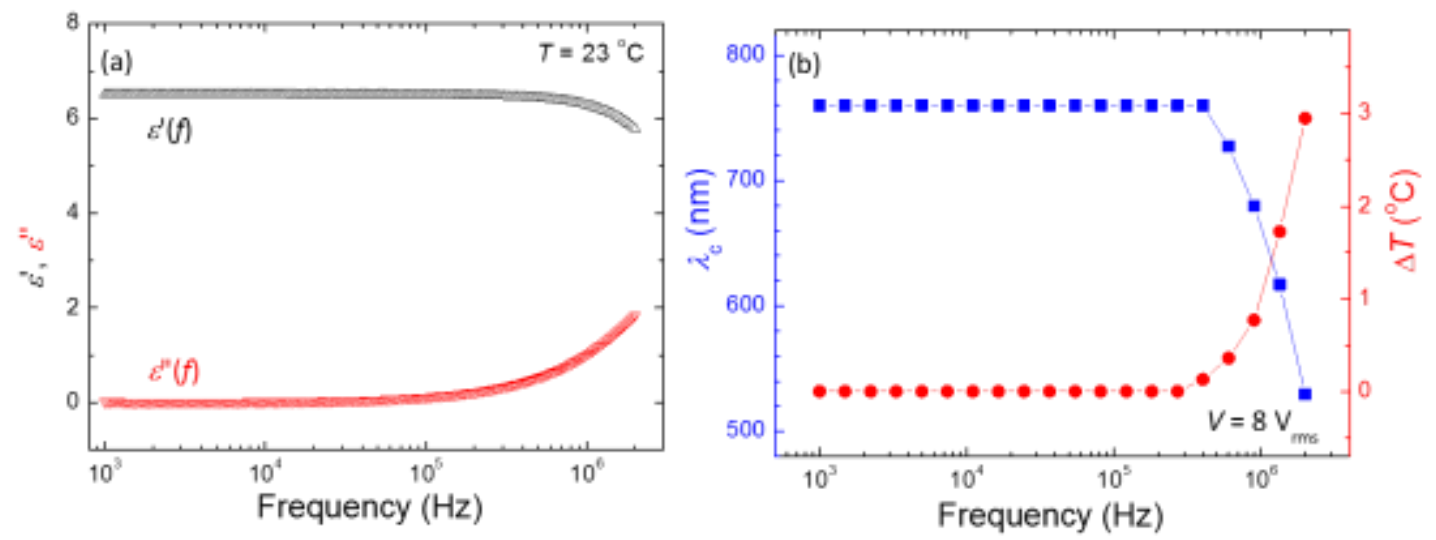

Figure 5. (a) Complex dielectric spectra of the CLC cell-A with $R_{\mathrm{ITO}}=110 \Omega$ at $T=23{ }^{\circ} \mathrm{C}$ and (b) frequency-dependent $\lambda_{\mathrm{c}}$ and $\Delta T$ of this cell driven by $8-\mathrm{V}_{\mathrm{rms}}$ voltages.

$\lambda_{\mathrm{c}}(T)=\gamma\left(\frac{T_{0}}{T}\right)\left(1+\frac{\beta}{T-T_{\mathrm{SmA}}}\right)^{2}$

with the fitting parameters $\gamma=321.58 \mathrm{~nm}$ and $\beta=1.08 \mathrm{~K}$, it is reported that the tunable range of $\lambda_{\mathrm{c}}$ at temperatures in the CLC phase of the cell could be very wide, ranging from $1392 \mathrm{~nm}$ (infrared) at $22{ }^{\circ} \mathrm{C}$ to $340 \mathrm{~nm}$ (ultraviolet) at $38^{\circ} \mathrm{C}^{26,27}$ Accordingly, the combination of or interplay between this thermally responsive CLC and pseudo-dielectric heating is unique, enabling the tunability of $\lambda_{\mathrm{c}}$ or reflective colors by applying external AC voltages to elevate the cell temperature. Figure 3 demonstrates the change in reflective optical texture and illustrates some transmission spectra in the visible light range of the CLC cell at a fixed frequency of $f=2 \mathrm{MHz}$ to evaluate the feasibility of the above-mentioned electro-thermal technique. In this experiment, the cell temperature in the field-off state is maintained at $T=22^{\circ} \mathrm{C}$ so that the corresponding optical texture reveals dark appearance due to reflection in the infrared. With increasing voltage, the reflective color of the cell changes from no color in the near infrared at $V=3.5 \mathrm{~V}_{\mathrm{rms}}\left(\lambda_{\mathrm{c}} \sim 800 \mathrm{~nm}\right)$, to red at $V=4.5 \mathrm{~V}_{\mathrm{rms}}\left(\lambda_{\mathrm{c}} \sim 700 \mathrm{~nm}\right)$, orange at $V=5.5 \mathrm{~V}_{\mathrm{rms}}\left(\lambda_{\mathrm{c}} \sim 600 \mathrm{~nm}\right)$, green at $V=6.5 \mathrm{~V}_{\mathrm{rms}}\left(\lambda_{\mathrm{c}} \sim 540 \mathrm{~nm}\right)$, blue at $V=7.5 \mathrm{~V}_{\mathrm{rms}}\left(\lambda_{\mathrm{c}} \sim 480 \mathrm{~nm}\right)$, navy at $V=9 \mathrm{~V}_{\mathrm{rms}}\left(\lambda_{\mathrm{c}}\right.$ $\sim 440 \mathrm{~nm}$ ), and to violet at $V=12 \mathrm{~V}_{\mathrm{rms}}\left(\lambda_{\mathrm{c}} \sim 380 \mathrm{~nm}\right)$, leading to the observed blueshift of the CLC bandgap in the transmission spectra. As the voltage goes higher than $12 \mathrm{~V}_{\mathrm{rms}}$, the center wavelength of the bandgap is thought to appear in the ultraviolet regime, as evidenced by the dark appearance of the reflective optical texture at $V=15 \mathrm{~V}_{\text {rms. }}$.

Figure 4(a) summarizes the voltage-dependent $\lambda_{\mathrm{c}}$ of the cell at five frequency conditions. Here the temperature was set to $T=23{ }^{\circ} \mathrm{C}$ so that $\lambda_{\mathrm{c}}$ is $\sim 760 \mathrm{~nm}$ at $\mathrm{V}=0 \mathrm{~V}_{\mathrm{rms}}$ and the amount of $\lambda_{\mathrm{c}}$ variation $\left(\Delta \lambda_{\mathrm{c}}\right)$ is defined as $760 \mathrm{~nm}-\lambda_{\mathrm{c}}(V)$. In the case of $f=1 \mathrm{kHz}, \lambda_{\mathrm{c}}$ is invariant at voltages between $5 \mathrm{~V}_{\mathrm{rms}}$ and $12 \mathrm{~V}_{\mathrm{rms}}$, indicating that this frequency is too low to trigger pseudo-dielectric heating. Increasing the frequency sequentially from $1 \mathrm{kHz}$ to $1.3 \mathrm{MHz}$ widens the tunable $\lambda_{\mathrm{c}}$ range 
with $\Delta \lambda_{\mathrm{c}}$ promoted from $\Delta \lambda_{\mathrm{c}}=205 \mathrm{~nm}$ at $5 \mathrm{~V}_{\mathrm{rms}}$ to $\Delta \lambda_{\mathrm{c}}=376 \mathrm{~nm}$ at $12 \mathrm{~V}_{\mathrm{rms}}$. Furthermore, by substituting the results of Fig. 4(a) into Eq. (6) with parameters of $\gamma=321.58 \mathrm{~nm}$ and $\beta=1.08 \mathrm{~K}$ to deduce the corresponding cell temperatures, one can identify from Fig. 4(b) that $\Delta T$ is linearly proportional to the square of voltage $\left(\Delta T \propto V^{2}\right)$ at frequencies higher than $f_{0}$, exactly following Eq. (3). Consequently, it is suggested that both the amplitude and frequency are responsible as variables for tuning CLC bandgap based on the proposed electro-thermal technique.

Finally, we attempt to inject the same CLC mixture (i.e., MLC-6608 + $45 \mathrm{wt} \%$ S-811) into another planar-aligned empty cell to prove that the above results predicted by Eq. (5) are non-specific. The parameters of this empty cell, designated cell-R, are $A=0.25 \mathrm{~cm}^{2}, d=5 \mu \mathrm{m}$, and $R_{\text {Iто }} \sim 110 \Omega$. It is displayed in Fig. 5(a) that the profile of the pseudodielectric relaxation is not as obvious as that in Fig. 2(a). This is due to the use of a cell with a smaller electrode area (of $0.25 \mathrm{~cm}^{2}$ compared with $1 \mathrm{~cm}^{2}$ ) and $R_{\mathrm{ITO}}$ (of $110 \Omega$ in comparison with $310 \Omega$ ) that significantly shifts $f_{\mathrm{PR}}$ to a frequency beyond the investigated frequency regime (between $1 \mathrm{kHz}$ and $2 \mathrm{MHz}$ determined by the instrumental limitation), conforming to Eq. (1). To evaluate the $\lambda_{\mathrm{c}}$ tuning ability, Fig. 5(b) delineates the frequency-dependent $\lambda_{\mathrm{c}}$ and $\Delta T$ of cell-R under the application of $8-\mathrm{V}_{\mathrm{rms}}$ voltages. One can still perform electrically tunable $\lambda_{\mathrm{c}}$ using cell-R but the tunable range (between $760 \mathrm{~nm}$ at $1 \mathrm{kHz}$ and $529 \mathrm{~nm}$ at $2 \mathrm{MHz}$ ) by $8-\mathrm{V}_{\text {rms }}$ voltage is narrower than that of the CLC with $R_{\text {ITO }} \sim 310 \Omega$ (between $760 \mathrm{~nm}$ at $1 \mathrm{kHz}$ and $448 \mathrm{~nm}$ at $2 \mathrm{MHz}$ ) by $V=8 \mathrm{~V}_{\text {rms }}$ (Fig. 4(b)). This finding results from the weakened dielectric heating efficacy and, thus, insufficient increase in temperature of cell-R with lowered $f_{\mathrm{R}}$ under voltage applied. Besides, experiments using cells with various sets of cell parameters have also been carried out in our recent works. ${ }^{26-28}$ It is concluded that the cell gap, the electrode area and the intrinsic dielectric anisotropy in LC used are responsible for the measured dielectric spectrum, thereby determining the profile of the pseudo-dielectric relaxation and the strength of the dielectric heating effect. For example, according to Eqs. (3) and (5), using a cell with low electrode resistivity and specific heat conductivity can enlarge the maximal tunable temperature range while appropriately adjusting the electrode area, cell gap and inherent dielectric permittivity of the LC can be in favor of lowering the voltage frequency and reducing the voltage amplitude needed for desired electro-thermal tuning range for the center wavelength by means of the externally controlled pseudo-dielectric relaxation.

\section{CONCLUSION}

The pseudo-dielectric relaxation is an unavoidable "false" signal in the complex dielectric spectrum of a sandwichtype LC cell with finite electrode conductivity. Many dielectric studies misinterpret the artifact as a property of the dielectric medium confined in an ITO cell regarded as a parallel-plate capacitor. Experienced researchers when performing dielectric investigations on LC cells generally consider this artefact as a nuisance and tend to suppress or exclude it. In this study, we have confirmed that the pseudo-dielectric relaxation can become advantageous and it is utilizable for the induction of heating, leading to the elevation in cell temperature by an externally applied AC voltage. Distinct from the well-known dielectric heating effect originating in the molecular rotation, the observed pseudo-dielectric relaxation is attributable to the pseudo-dielectric energy losses from the cell geometry contributing to the resistance $\left(R_{\text {ITO}}\right)$-capacitance $\left(C_{\mathrm{LC}}\right)$ circuit. The underlying mechanism and factors governing the pseudo-dielectric heating efficiency have theoretically been interpreted by a modified model. ${ }^{22}$ Our finding indicates that the dielectric heating is no longer the feature specific to DFLCs but is applicable for all LC materials. Furthermore, we have demonstrated a novel approach for electro-thermally tuning the central wavelength $\left(\lambda_{c}\right)$ of the CLC bandgap by combining this unique pseudo-dielectric heating effect with a thermo-sensitive $-\Delta \varepsilon$ CLC. The adopted CLC mixture is characterized by its widely tunable $\lambda_{c}$ in the infrared, visible and ultraviolet wavelength ranges by temperature variations. Upon the application of AC voltage to the cell, our results suggest that the reflective color can be directly and reversibly modulated by $2 \mathrm{MHz}$ voltages from infrared at $V=0 \mathrm{~V}_{\text {rms }}$, across the visible spectral range at $3.5 \mathrm{~V}_{\text {rms }}<\mathrm{V}<12 \mathrm{~V}_{\text {rms }}$, and to ultraviolet at $V=15 \mathrm{~V}_{\text {rms. }}$. From the point of view of ease of fabrication, the proposed negative CLC cell is simple because all components used, including the empty sandwich-type cells with ITO-coated substrates and LC materials, are commercially available. In contrast to other electrical tuning methods, this study provides a new pathway to a wider tunable reflective color range with lower operating voltage, promoting the applicability of CLC for the design of superior color-reflective displays and electrically tunable photonic devices.

\section{ACKNOWLEDGMENTS}

This study is financial supported by the Ministry of Science and Technology, Taiwan, under grant Nos. 104-2112-M-009008-MY3, 106-2923-M-009-002-MY3, and 107-2112-M-009-012-MY3. 


\section{REFERENCES}

[1] D. Broer, J. Lub, and G. Mol, "Wide-band reflective polarizers from cholesteric polymer networks with a pitch gradient," Nat., 378, 467-469 (1995).

[2] D. Coates, "Development and applications of cholesteric liquid crystals," Liq. Cryst., 42, 653-665 (2015).

[3] D.-K. Yang, J. L. West, L.-C. Chien, and J. W. Doane, "Control of reflectivity and bistability in displays using cholesteric liquid crystals," J. Appl. Phys. 76, 1331-1333 (1994).

[4] N. Y. Ha, Y. Ohtsuka, S. M. Jeong, S. Nishimura, G. Suzaki, Y. Takanishi, K. Ishikawa, and H. Takezoe, "Fabrication of a simultaneous red-green-blue reflector using single-pitched cholesteric liquid crystals," Nat. Mater. 7, 43-47 (2008).

[5] S. J. Woltman, G. D. Jay, and G. P. Crawford, "Liquid-crystal materials find a new order in biomedical applications," Nat. Mater., 6, 929-938 (2007).

[6] Y.-C. Hsiao, Y.-C. Sung, M.-J. Lee, and W. Lee, "Highly sensitive color-indicating and quantitative biosensor based on cholesteric liquid crystal," Biomed. Opt. Express, 6, 5033-5038 (2015).

[7] T.-H. Lin, C.-H. Chen, Y. Chen, T. Wei, C.-W. Chen, and A. Y.-G. Fuh, "Electrically controllable laser based on cholesteric liquid crystal with negative dielectric anisotropy," Appl. Phys. Lett. 88, 061122 (2006).

[8] L. V. Natarajan, J. M. Wofford, V. P. Tondiglia, R. L. Sutherland, H. Koerner, R. A. Vaia, and T. J. Bunning, "Electro-thermal tuning in a negative dielectric cholesteric liquid crystal material," J. Appl. Phys. 103, 093107 (2008).

[9] C. A. Bailey, V. P. Tondiglia, L. V. Natarajan, M. M. Duning, R. L. Bricker, R. L. Sutherland, T. J. White, M. F. Durstock, and T. J. Bunning, "Electromechanical tuning of cholesteric liquid crystals," J. Appl. Phys. 107, 013105 (2010).

[10] S. S. Choi, S. M. Morris, W. T. S. Huck, and H. J. Coles, "Wavelength tuning the photonic band gap in chiral nematic liquid crystals using electrically commanded surfaces," Appl. Phys. Lett. 91, 231110 (2007).

[11] S. S. Choi, S. M. Morris, W. T. S. Huck, and H. J. Coles, "Electrically tuneable liquid crystal photonic bandgaps,"Adv. Mater. 21, 3915-3918 (2009).

[12] J. Xiang, Y. Li, Q. Li, D. A. Paterson, J. M. D. Store, C. T. Imrie, and O. D. Lavrentovich, "Electrically tunable selective reflection of light from ultraviolet to visible and infrared by heliconical cholesterics," Adv. Mater. 27, 3014-3018 (2015).

[13] P. N. Keating, "A theory of the cholesteric mesophase," Mol. Cryst. Liq. Cryst. 8, 315-326 (1969).

[14] S.-Y. T. Tzeng, C.-N. Chen, and Y. Tzeng, "Thermal tuning band gap in cholesteric liquid crystals," Liq. Cryst. 37, 1221-1224 (2010).

[15] F. Zhang and D.-K. Yang, "Temperature dependence of pitch and twist elastic constant in a cholesteric to smectic A phase transition," Liq. Cryst. 29, 1497-1501 (2002).

[16] Y. Huang, Y. Zhou, C. Doyle, and S.-T. Wu, "Tuning the photonic band gap in cholesteric liquid crystals by temperature-dependent dopant solubility," Opt. Express 3, 1236-1242 (2006).

[17] Y. Wang, Z. Zheng, H. K. Bisoyi, K. G. Gutierrez-Cuevas, L. Wang, R. S. Zola, and Q. Li, "Thermally reversible full color selective reflection in a self-organized helical superstructure enabled by a bent-core oligomesogen exhibiting a twist-bend nematic phase," Mater. Horizons 3(5), 442-446 (2016).

[18] Z. Cheng, K. Li, R. Guo, F. Wang, X. Wu, L. Zhang, J. Xiao, H. Cao, Z. Yang, and H. Yang, "Bandwidthcontrollable reflective polarisers based on the temperature-dependent chiral conflict in binary chiral mixtures," Liq. Cryst. 38, 233-239 (2011).

[19] L. Wang, K. G. Gutierrez-Cuevas, A. Urbas, and Q. Li, "Near-infrared light-directed handedness inversion in plasmonic nanorod-embedded helical superstructure," Adv. Opt. Mater., 4(2), 247-251.

[20] Y. C. Hsiao, Z. H. Yang, D. Shen, and W. Lee, "Red, green, and blue reflections enabled in an electrically tunable helical superstructure," Adv. Opt. Mater., 6, 1701128 (2018).

[21] P. Perkowski, D. Lada, K. Ogrodnik, J. Rutkowska, W. Piecek, and Z. Raszewski, "Technical aspects of dielectric spectroscopy measurements of liquid crystals," Opto-Electron. Rev. 16, 271-276 (2008).

[22] M. Schadt, "Dielectric heating and relaxations in nematic liquid crystals," Mol. Cryst. Liq. Cryst. 66, 319-336 (1981).

[23] C.-H. Wen and S.-T. Wu, "Dielectric heating effects of dual-frequency liquid crystals," Appl. Phys. Lett. 86, 231104 (2005). 
[24] Y. Yin, S. V. Shiyanovskii, and O. D. Lavrentovich, "Electric heating effects in nematic liquid crystals," J Appl. Phys. 100, 024906 (2006).

[25] P. Perkowski, "How to determine parameters of soft mode from dielectric spectroscopy performed using cells with ITO electrodes?" Opto-Electron. Rev. 19, 76-82 (2011).

[26] P.-C. Wu, G.-W. Wu, I. V. Timofeev, V. Y. Zyryanov, and W. Lee, "Electro-thermally tunable reflective colors in a self-organized cholesteric helical superstructure," Photonics Res. 6(12), 1094-1100 (2018).

[27] P.-C. Wu, G.-W. Wu, C.-H. Yu, and W. Lee, "Voltage-induced pseudo-dielectric heating and its application for color tuning in a thermally sensitive cholesteric liquid crystal," Liq. Cryst. (2019), https://doi.org/10.1080/02678292.2019.1641753

[28] C.-H. Yu, P.-C. Wu and W. Lee, "Electro-thermal formation of uniform lying helix alignment in a cholesteric liquid crystal cell," Cryst. 9(4), 183-1-10 (2019). 